\title{
DIFFERENT SURGICAL TECHNIQUES OF SKULL BASE RECONSTRUCTION: EVALUATION AND OUTCOME
}

Hazem S. Soliman, Samy H. Mohamed, Tarek H. Abdel-Bary and Mohamed Reda Abd Elaziz

Neurosurgery departments, Faculty of Medicine,Zagazig University

\begin{abstract}
Background : cerebrospinal fluid (CSF) leak is the most common complication after skull base surgery. It carries a high risk of life threatening complications (e.g., tension pneumocephaly, meningitis, cerebritis, and brain abscess).

Patients and Methods: We present twenty patients, ages 2-55 years, eight males and twelve females with different skull base pathologies operated by different surgical modalities (microscopic transcranial, endoscopic endonasal and combined micro-endoscopic techniques) at neurosurgery department, faculty of medicine, Zagazig University, between Jan. 2016 to Jan. 2018. Prophylactic antibiotics and anticonvulsants were rourtinely given. Lumbar drain was not used.

Results: the success rate was $(95 \%)$ and only one patient $(5 \%)$ developed post operative CSF leak which responded to conservative measures. This suggests that proper sealing of the skull base defect has been achieved. There was no infection, no mortality and the long term follow up showed good functional and aesthetic results.

Conclusion: The reconstructive process although being the last step in the surgical procedure, but it's a very important one. It should be planned well before surgery. Meticulous repair of the already present or resultant defect should be performed in every case.

Key words: Skull base defect, skull base reconstruction, cerebrospinal fluid leakage, dural reconstruction.

Corresponding author: Mohamed Reda Abd Elaziz

Mohamed .reda.neuro@gmail.com

01006345859
\end{abstract}

\section{INTRODUCTION}

$S^{\prime}$ urgical procedures involving the skull base are considered the most difficult in neurosurgery. The efficacy of any surgical procedure for skull base tumour resection is also determined by the ability to repair the resulting defect, which has been a challenging task over the past decade. 2

The primary goal of skull base reconstruction is to provide a watertight separation of the cranial cavity from the sinonasal tract to prevent cerebrospinal fluid [CSF] leak, thereby decreasing the risk of devastating sequelae [e.g. pneumocephalus, meningitis]. Other goals include protection of neurovascular structures obliteration of the dead space and achievement of good functional and cosmetic outcome. 3

There are various skull base pathologies which necessitate reconstruction. They can be broadly classified into congenital [e.g. encephaloceles], traumatic fracture base (e.g. accidental, iatrogenic), inflammatory [e.g. sinonasal infections with intracranial extension), neoplastic (e.g. skull base tumours, sinonasal tumours eroding the base of the anterior or middle cranial fossae] and idiopathic (e.g. idiopathic/spontaneous CSF leaks). 4
The first surgical repair of a postoperative dural defect was reported in 1913; the dural defect was sutured and reinforced with canine allograft. Thirty years later, Dandy corrected these defects with fascia. Subsequently various techniques were used using a variety of natural and synthetic materials (e.g. pericranial flaps, fascia lata grafts, mucosal grafts, fat, cartilage, bone, synthetic dura and titanium mesh), beside the use of tissue sealants [e.g. fibrin glue and histoacryl].5

Various surgical modalities have been described for reconstruction including open (transcranial). endoscopic (endonasal] and endoscopic assisted approaches.6

A reconstructive plan must always be tailored for the individual patient. It is therefore imperative that the skull base surgeon be comfortable with the different reconstructive methods. 7

The choice of specific reconstructive technique depends upon many factors e.g.

- Site and size of the skull base defect.

- The condition of the surrounding dura and bone.

- Extent of communication between the nasal and intracranial cavities.

- The nature of the lesion resected. 
- Patient comorbidities [e.g. diabetes mellitus, immuno-suppression).

- Status of the nasal septum and lateral nasal wall.

- History of previous surgery or radiotherapy.

- The anticipated need for adjuvant therapies [i.e. irradiation or

\section{Chemotherapy].8}

\section{PATIENTS \& METHODS}

After institutional review board approval, we conducted a prospective study of a consecutive cohort of patients undergoing surgery for a variety of skull base pathologies. During the period from Jan. 2016 to Jan. 2018 at neurosurgery department, Faculty of Medicine, Zagazig University, 20 consecutive patients were identified. The patients included 8 males and 12 females with an age range of 2 to 55 years (median: 31 ). Preoperative work up included computed tomography (axial, cornoal, sagittal and 3D reconstruction images) and MR imaging. B2 transferin was not searched for. Intrathecal injection of flourescin to localize the site of the defect was done in one patient.

\section{RESULTS}

20 patients with different skull base pathologies were included in the study. The commonest etiology was neoplastic lesions, followed by traumatic fracture base, while congenital lesions and spontaneous CSF rhinnorhea were the least common (Fig. 1).

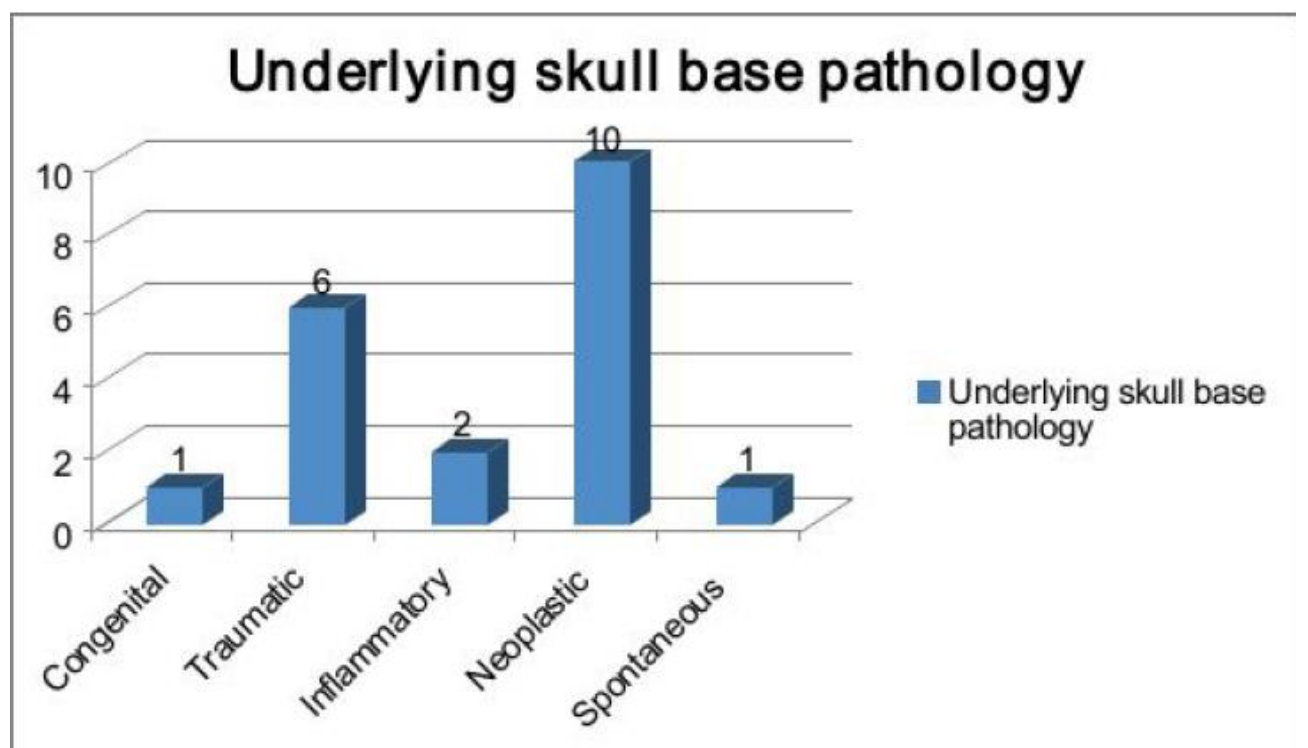

Cribriform plate of the ethmoid bone was the commonest skull base defect followed by the sellar floor, while the posterior wall of the frontal sinus was the least common (Fig. 2).

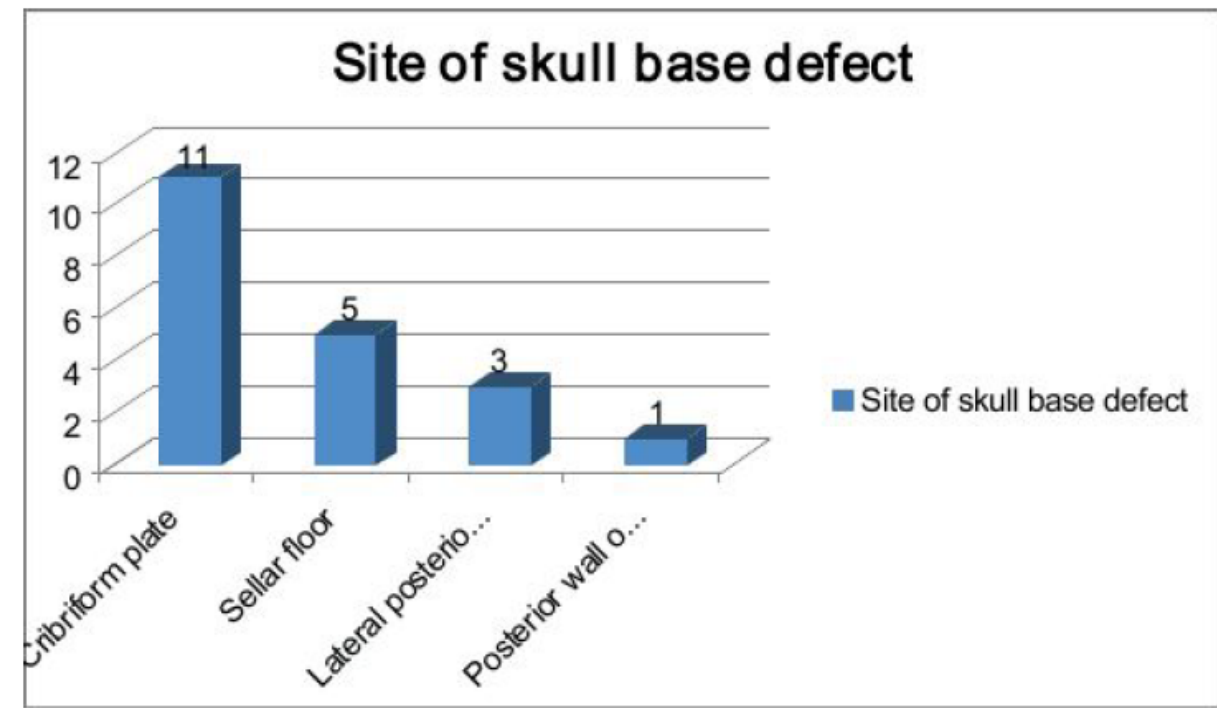

In one third of cases, the defect was single, while multiple defects were found in two third of cases (Fig. 3). 


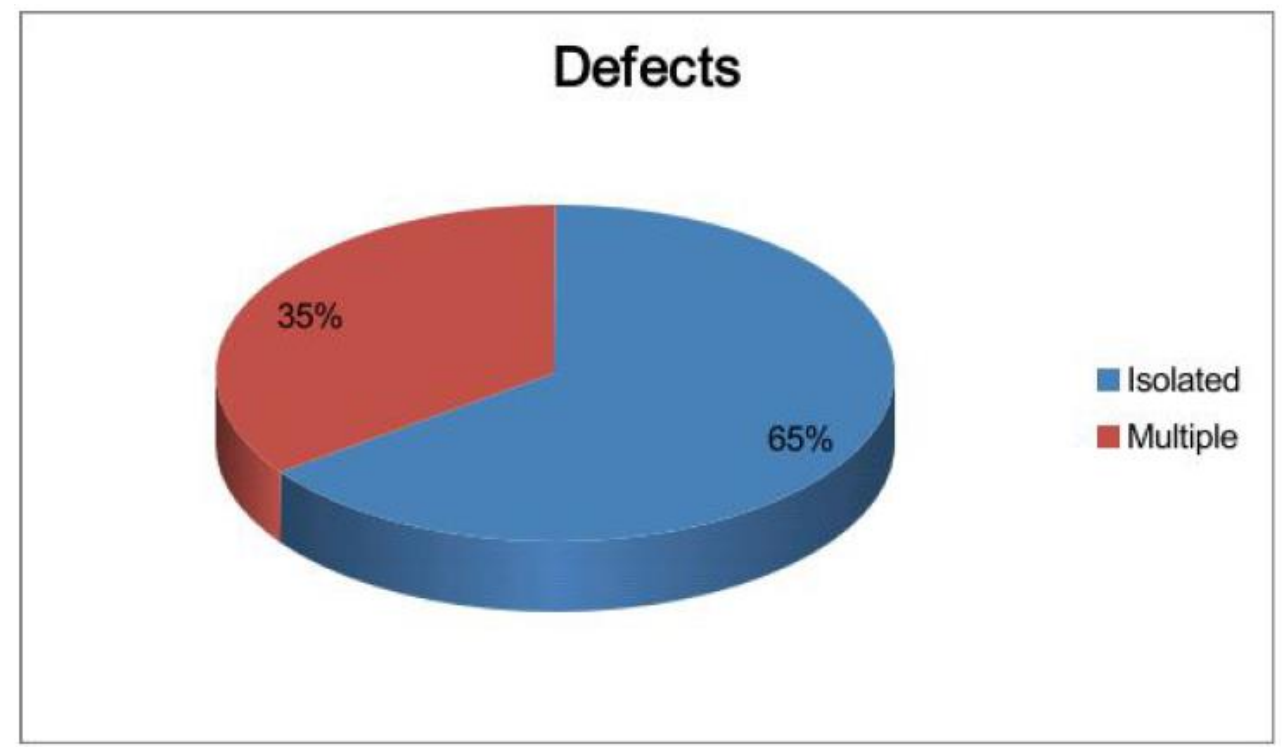

Different techniques were used to repair the underlying defect: Open (transcranial 50\%), pure endoscopic endonasal 40\%, and combined (endoscopic assisted open microsurgery 10\%) (Fig. 4).

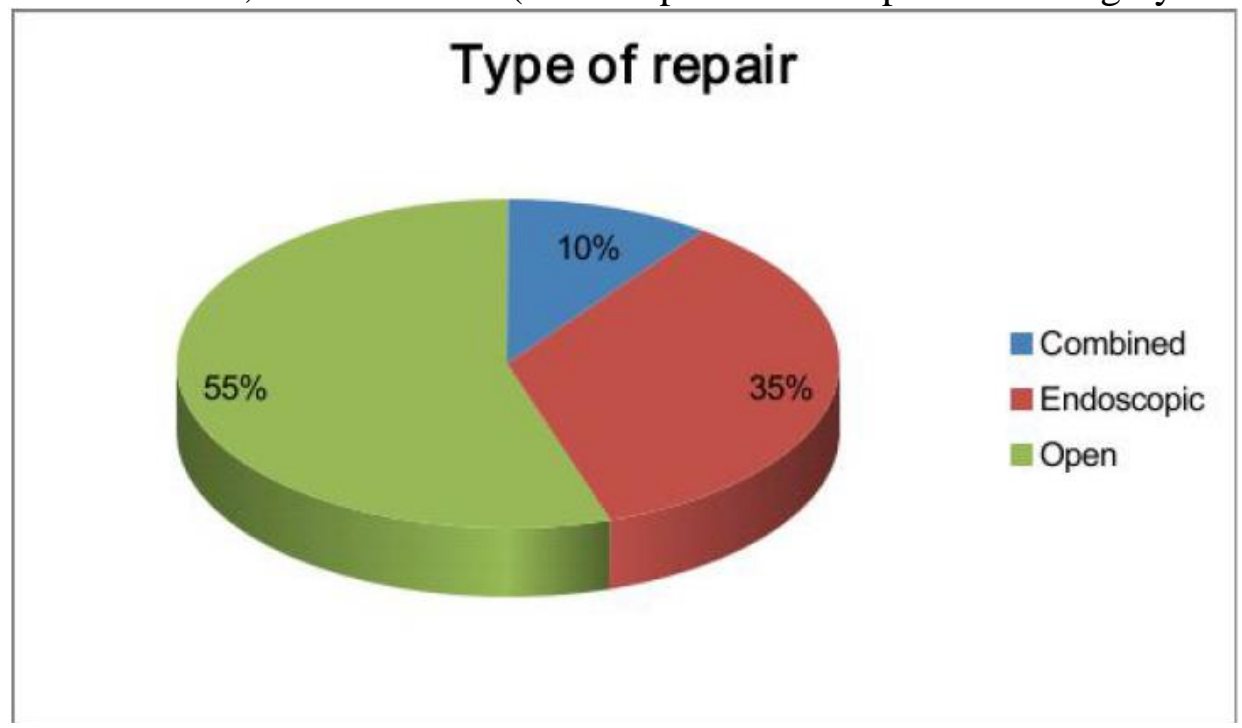

Bi-frontal approach was used in 7 (35\%) patients, retrosigmoid approach was used in $3(15 \%)$ patients, pure endoscopic endonasal approach was used as a single procedure in $8(40 \%)$ patients, while combined cranio-endoscopic technique was used in $2(10 \%)$ patients (Table 1).

\begin{tabular}{|l|c|c|}
\hline & $\mathrm{N}$ & $\%$ \\
\hline Bi-frontal approach & $\mathbf{8}$ & $\mathbf{4 0 . 0} \%$ \\
\hline Endoscopic endo-nasal & $\mathbf{7}$ & $\mathbf{3 5 . 0} \%$ \\
\hline Retro-sigmoid approach & $\mathbf{3}$ & $\mathbf{1 5 . 0} \%$ \\
\hline Combined cranio-endoscopic & $\mathbf{2}$ & $\mathbf{1 0 . 0} \%$ \\
\hline
\end{tabular}

Different materials (natural and synthetic) were used in the reconstructive process (Table 2). 


\begin{tabular}{|l|l|l|l|}
\hline \multirow{2}{*}{ Natural } & Mucosal graft & N & $\%$ \\
\cline { 2 - 3 } & G.P & $\mathbf{3}$ & $\mathbf{1 5 \%}$ \\
\hline Fascia lata & $\mathbf{2}$ & $\mathbf{3 5 \%}$ \\
\hline \multirow{5}{*}{ Synthetic } & Fat & $\mathbf{2}$ & $\mathbf{1 0} \%$ \\
\cline { 2 - 3 } & Bone ships & $\mathbf{8}$ & $\mathbf{4 0} \%$ \\
\hline & Gel foam & $\mathbf{5}$ & $\mathbf{2 5 \%}$ \\
\cline { 2 - 4 } & Jurgicele & $\mathbf{1}$ & $\mathbf{5 \%}$ \\
\hline & Acrylic & $\mathbf{1}$ & $\mathbf{5 \%}$ \\
\hline
\end{tabular}

The overall success rate was $95 \%$. Only one patient developed postoperative CSF leak which responded to conservative measures. None of the patients developed meningitis, brain abscess or tension pneumocephalus.

\section{DISCUSSION}

Reconstruction is an integral part of skull base surgery and planning should always begin with the decision to perform surgery. The basic concept of reconstruction is the restoration of the skull base integrity to avoid the communication between the "sterile" intracranial cavity and the "contaminated" sinonasal tract with its devastating sequelae(1).

The most common and the most scared complication following skull base surgery is: CSF leakage, which carries a high risk of pneumocephalus, meningitis, cerebritis and brain abscess(9).

In our study, the etiological examination of the patients revealed that the most frequently observed reason was tumoral lesions $(50 \%)$, followed by cranial traumas (30\%). This finding can be explained by the fact that our hospital is "atertiary referral" hospital with all the facilities for performing such challenging skull base tumors are already available at our department.

In our study, the commonest site of skull base defect was the cribriform plate of ethmoid bone $(55 \%)$ and the least common site was the posterior wall of the frontal air sinus (5\%). In agreement with our results, Isam Al Obid et al., (2014), in their series of skull base reconstruction reported that the cribriform plate of ethmoid bone was the commonest site of defect $(50 \%)$, whilst the frontal sinus was the least common site (4.8\%) (10).

Many techniques can be used to repair the underlying skull base defect. We used different surgical modalities in our study: Open "trans-cranial" (50\%), pure endoscopic endonasal (40\%) and combined craniondoscopic approaches (10\%). In our study, both natural and synthetic materials were used to achieve good reconstruction of the skull base defects (e.g., pericranial flap, middle turbinate mucosal graft, fascia lata, fat, bone chips and polymethyl methacrylate).

In agreement with our technique of reconstruction, Jesse and Yadranko, (2004), used the pericranial flap for closure of different skull base defects in 32 patients during the period from September 1997 to July 2003. They reported 2 cases of transient CSF leak without resultant meningitis, which resolved without surgical intervention or the placement of lumbar drain (11).

Ziv et al., (2009), used fascia lata for reconstruction of extensive post traumatic anterior skull base defects in (9) children. Fibrin glue was used to provide additional protection against CSF leak. None of the operated children developed CSF leak or meningitis(12). We used 2 different materials for reconstructing the subocinital craniectomy defect, autologous bone clips (5\%) and polymethyl methacrylate (10\%) with good postoperative functional and cosmetic outcome.

Missori et al., (2002), agree with our results. They used bone chips for reconstructing the suboccipital defect in 15 patients (13).

Also, Doo et al., (2007), used polymethyl methacrylate for reconstructing the suboccipital craniectomy defect in a large 
series of patients (678) during 10 years period (Jan. 1996 to Feb. 2006).

They reported only 2 cases $(0.29 \%)$ of post operative CSF leak which were treated with lumbar drain. No postoperative infection or allergy from acrylic was noted (14).

Chris et al., (2015), also agree with our technique of repair. They used natural materials (abdominal fat, septal bone) in 235 patints during the period from 2005 to 2012.4 patients $(1.7 \%)$ developed postoperative CSF leak which were treated with lumbar drain(15).

\section{CONCLUSION}

Meticulous reconstruction of the skull base defects appears to decrease the risk of CSF leakage and other life threatening complications. Natural materials (e.g., pericranial flap, fascia lata, fat, ...) are already available without cost and can achieve reliable reconstruction.

\section{Conflicts of interest: None}

\section{REFERENCES}

1. Treasure, Trevor E. Jeffrey S. Dean and Robert D. Gear. "Craniofascial approaches and reconstruction in skull base surgery: techniques for the oral and maxillofacial surgeon". Journal of Oral and Maxillofacial Surgery, 71.12 (2013): 2137-2150.

2. Paolo C, Andrea P and Stefania G (2015): Dural reconstruction. Endonasal endoscopic surgery of skull base tumors: an interdisciplinary approach, Thieme Medical Publisher, 284-296.

3. Klatt-Cromwell C et al., (2016): Reconstruction of skull base defects. Otolaryngologic Clinics of North America 49.1: 107-117.

4. Woodworth B and Rodney J (2007): Repair of anterior skull base defects and CSF leaks. Rhinologic and sleep apnea surgical techniques. Springer Berlin Heidelberg, 139147.

5. Khan A, Ari L and David J. (2014): Use of titanium mesh for middle cranial fossa skull base reconstruction. Journal of neurological surgery. Part B, skull base 75.2: 104.

6. Chaaban Mohamed and Bradford A. (2012): Complications of skull base reconstruction. 148-162.

7. Rawal, Rounak et al. (2013): Advances in reconstruction of the skull base. Current Otorhinolaryngology Reports 1.4: 191-196.
8. Campbell, Rawnak et al. (2016): Cranial base reconstruction after transcranial and transnasal skull base surgery for median lesions. Midline skull base surgery. Springer international publishing, 333-362.

9. Murat G, Ibrahim E, Mehmet A et al., (2016): Anterior skull base defects reconstructed using three-layer method: 78 consecutive cases with long-term follow-up. J Neurol Surg, 77: 499-502.

10. Isam A, Joaquim E, Elena R et al., (2013): Management of cerebrospinal fluid leaks according to size. Our experience, Acta Otorhinolaringology Esp, 65(3): 162-169.

11. Jesse E and Yadranko D (2004): The versatile extended pericranial flap for closure of skull base defects. Otolaryngol Head Neck Surg, 130: 704-11.

12. Zic G, Gadi F, Dan M et al., (2009): Multidisciplinary surgical approach for cerebrospinal fluid leak in children with complex head trauma. Childs Nerv Syst, 25: 915-923

13. Missori P, Rastelli E, Polli F et al., (2002): reconstruction of suboccipital craniectomy with autologous bone chips. Acta Neurochir, 144: 917-920.

14. Doo SK, Jae S, Jeong A et al., (2007): Intraoperative management to prevent cerebrospinal fluid leakage after microvascular decompression: dural closure with a "plugging muscle" method. Neurosurg Rev, 30: 139-142.

15. Crhis ST, Amjad A, Jennifer $\mathrm{K}$ et al., (2015): Sellar reconstruction and rates of delayed cerebrospinal fluid leak after endoscopic pituitary surgery. J Neurol Surg B, 76: 281-285. 